\title{
Myeloid expression of angiotensin-converting enzyme facilitates myeloid maturation and inhibits the development of myeloid-derived suppressor cells
}

\author{
Xiao Z Shen ${ }^{1,2}$, Derick Okwan-Duodu ${ }^{1,3}$, Wendell-Lamar Blackwell ${ }^{1}$, Frank S Ong ${ }^{1}$, Tea Janjulia ${ }^{1}$, Ellen A Bernstein ${ }^{1}$, \\ Sebastien Fuchs ${ }^{1,2}$, Serhan Alkan ${ }^{2}$ and Kenneth E Bernstein ${ }^{1,2}$
}

Myeloid-derived suppressor cells (MDSCs) are a heterogeneous population of immature myeloid cells which accumulate in cancer, infection and chronic inflammation. These cells suppress T-cell function and the immune response.

Angiotensin-converting enzyme (ACE) is a peptidase that is now known to regulate aspects of myelopoiesis. Here, we show that ACE expression correlates with myeloid maturation in vitro. Forced ACE overexpression in monocytic cells reduces the generation of MDSCs. In vivo, mice with a genetic change resulting in myeloid cell ACE overexpression have reduced numbers of blood and splenic MDSCs in a tumor model and in a model of chronic inflammation induced by complete Freund's adjuvant. In contrast, ACE-null mice produce large numbers of MDSCs during chronic inflammation. Macrophages from mice with myeloid ACE overexpressing are more pro-inflammatory and have more tumor-killing activity than cells from wild-type mice. Thus, manipulating myeloid ACE activity can interfere with MDSC development and the maturation of myeloid cells.

Laboratory Investigation (2014) 94, 536-544; doi:10.1038/labinvest.2014.41; published online 10 March 2014

KEYWORDS: angiotensin-converting enzyme; macrophages; myeloid-derived suppressor cells

Angiotensin-converting enzyme (ACE) is part of the reninangiotensin system, a cascade of enzymes and peptides traditionally associated with the regulation of blood pressure. ACE is a carboxypeptidase that converts the inactive precursor angiotensin I to the vasoconstrictor angiotensin II. ACE also cleaves a variety of other peptide substrates, including bradykinin and substance P. Perhaps because of its wide substrate specificity, ACE affects many different physiological processes. ${ }^{1}$

Recently, several groups have presented evidence detailing a role for ACE and angiotensin II in the immune response. For example, ACE and angiotensin II were shown to have a major role in a mouse model of experimental autoimmune encephalitis. ${ }^{2}$ Other studies showed that ACE affects normal myeloproliferation, myelodifferentiation and macrophage functional maturation. ${ }^{3,4}$

An important concept in immunology is that the inflammatory response is critical to contain infection, but it must be limited and ultimately terminated to avoid collateral injury. Mechanisms controlling inflammation are diverse and include anti-inflammatory cytokines, cytokine receptor antagonists and inhibitors of cytokine signaling. Further, at the cellular level, professional immuno-regulatory $\mathrm{CD}^{+}$ Foxp $3^{+} \mathrm{T}$ (Treg) cells have been identified. ${ }^{5}$ Another suppressor cell population is myeloid-derived suppressor cells (MDSCs), which are distinct from Tregs. ${ }^{6}$ MDSCs are comprised of a heterogeneous population of myeloid precursors, including precursors of macrophages, granulocytes and dendritic cells. MDSCs were first associated with tumor progression, and accumulation of such cells was reported in both mice and humans carrying tumors. ${ }^{7,8}$ Detailed characterization has demonstrated the ability of MDSCs to suppress several aspects of the immune response, especially T-cell functions. ${ }^{9}$ Although MDSCs were originally implicated in creating an immunosuppressive environment associated with tumor growth, it is now known that MDSCs can be induced

\footnotetext{
${ }^{1}$ Division of Immunology, Department of Biomedical Science, Cedars-Sinai Medical Center, Los Angeles, CA, USA; ${ }^{2}$ Department of Pathology and Laboratory Medicine, Cedars-Sinai Medical Center, Los Angeles, CA, USA and ${ }^{3}$ School of Medicine, Emory University, Atlanta, GA, USA

Correspondence: Dr KE Bernstein, MD, Division of Immunology, Department of Biomedical Science, Cedars-Sinai Medical Center, 110 N. George Burns Road, Davis 2021, Los Angeles, CA 90048, USA.

E-mail: kbernst@cshs.org
}

Received 28 July 2013; revised 30 December 2013; accepted 21 January 2014 
by a variety of inflammatory pathologies, including sepsis, trauma and chronic inflammation. ${ }^{10-12}$

In mice, MDSCs are defined as cells that simultaneously express the surface markers CD11b and Gr $1 .{ }^{13}$ More recently, MDSCs were divided into two subsets based on their expression of Ly6C. ${ }^{14}$ Ly6C ${ }^{\text {high }}$ cells have a monocytic-like morphology while Ly6 $\mathrm{C}^{\text {low/- }}$ cells have granulocytic-like morphology.

We and others have documented a role for ACE in myelopoiesis. ${ }^{3}$ Specifically, the lack of ACE results in the accumulation of immature myeloid precursors in the bone marrow (BM) and spleen. Here, we investigate the role of ACE in the development of MDSCs and present evidence that the expression of ACE in myeloid cells leads to the differentiation of these cells and limits the expansion of the suppressor MDSC population.

\section{MATERIALS AND METHODS Reagents}

Lipopolysaccharide (LPS) (Escherichia coli 055:B5) were from Sigma-Aldrich (St Louis, MO, USA). Granulocyte/macrophage colony-stimulating factor (GM-CSF) and IL-6 were purchased from Peprotech. Liposome clodronate were obtained from Dr Nico van Rooijen (Vrije Universiteit, Amsterdan, Netherlands).

\section{Mice}

Mice null of ACE and mice overexpressing myelomonocytic ACE (called ACE 10/10) have been previously described. ${ }^{15,16}$ Both lines were back-crossed at least 10 generations to C57BL/6 background. All mice were maintained in micro isolator cages, and all experimental protocols were approved by the Institutional Animal Care and Use Committee at Cedars Sinai Medical Center.

\section{Antibodies and Flow Cytometry}

The following antibody clones were used: GL1 (anti-CD86), 16-10A1 (anti-CD80), AF6-120.1 (anti-I-Ab), 145-2C11 (anti-CD3), M1/70 (anti-CD11b), BM8 (anti-F4/80), mIL4RM1 (anti-IL-4R), RB6-8C5 (anti-Gr1), 1A8 (anti-Ly6G), and HK1.4 (anti-Ly6C). All the above antibodies were purchased from either eBioscience, BioLegend (San Diego) or Pharmingen. The polyclonal rabbit-anti-ACE antibody was described previously. 7-Amino-actinomycin D (7-AAD) was from BioLegend. For examining MDSCs by flow cytometry, we used anti-CD11b vs 7-AAD to gate the live myeloid cells, followed by anti-Ly6C $v s$ anti-Ly6G staining to separate MDSC subsets. The stained samples were analyzed on a Beckman Coulter CyAn ADP, and data were analyzed by FlowJo software.

\section{In vitro Expansion of MDSCs}

Mice BM cells were obtained from the femurs and tibias. BM cells were cultured in RPMI 1640 medium supplemented with $10 \% \mathrm{FBS}, 25 \mathrm{ng} / \mathrm{ml} \mathrm{GM}-\mathrm{CSF}$ and either $40 \mathrm{ng} / \mathrm{ml}$ IL6 or $15 \%$
B16-F10 melanoma-conditioned media. The cultures were maintained at $37^{\circ} \mathrm{C}$ in a $5 \% \mathrm{CO}_{2}$-humidified atmosphere and after 4-day culture, cells were harvested and analyzed.

\section{In Vitro Immunosuppressive Assay}

Cells (MDSCs, macrophages or sorted subpopulations) were co-cultured with spleen-derived $\mathrm{T}$ cells at a ratio of $1: 1$ in the presence of anti-CD3 (Clone 145-2C11 from SouthernBiotech) and anti-CD28 (Clone 37.51 from SouthernBiotech) antibodies in a flat-bottomed plate. The $\mathrm{T}$ cells were pre-labeled with $2 \mu \mathrm{M}$ 5-(and 6)-carboxyfluorescein diacetate succinimidyl ester (Invitrogen). Three days later, cells were harvested, and $\mathrm{T}$ cells were identified by APC-labeled anti-CD4 and anti-CD8 antibodies with the incorporation of 7-AAD to exclude the dead cells. Cells were then subjected to flow cytometry analysis of 5-(and 6)-carboxyfluorescein diacetate succinimidyl ester profile.

\section{Melanoma Cells and Tumor Model}

The B16-F10 melanoma cell line American Type Culture Collection (ATCC, Manassas, VA, USA), were maintained as previously described. Tumor cell concentration was adjusted to $1 \times 10^{7} \mathrm{cells} / \mathrm{ml}$, and $100 \mu$ l of the suspension was injected intradermally into the dorsal skin. Tumor size was measured using a caliper, and volume was calculated according to the formula $\left(V=\left(L \times W^{2}\right) \times 0.52\right)$, where $V$ is the volume, $L$ is the length and $W$ the is width (length is greater than width). For lung metastasis, mice were injected intravenously with $5 \times 10^{5}$ B16-F10 cells in $100 \mu \mathrm{l}$ PBS. Fourteen days later, animals were killed, and dark nodules in the lungs were counted with the aid of a microscope.

\section{In Vivo Macrophage Depletion with Liposome Clodronate}

Macrophages were depleted by injection of $1 \mathrm{mg}$ of clodronate-encapsulated liposomes every $72 \mathrm{~h}$. Control mice were injected with liposomes containing PBS. Flow cytometry confirmed depletion of macrophages and other CD11b ${ }^{+}$ myeloid cells as previously reported.

\section{Thioglycollate-Elicited Peritoneal Macrophage (TPM) Isolation and Culture}

TPM were harvested from peritoneal cavity 3 days after 3\% thioglycollate i.p. injection and cultured as previously described. Macrophages were purified by cell culture dish adherence. In vitro killing assays were performed by seeding macrophages (effector) and tumor cells (target) at a 10:1 effector:tumor ratio. Some groups of effectors were primed with $100 \mathrm{ng} / \mathrm{ml}$ LPS overnight before co-culture with B16F10. After overnight incubation, tumor cell death was analyzed by an LDH release assay (Promega).

\section{Isolation of Macrophages from Tumor-bearing mice}

Mice with the establishment of B16 melanoma for 14 days were injected i.p. with $2 \mathrm{ml}$ aged thioglycollate broth. Four 
days later, peritoneal exudates cells were harvested. $\mathrm{F} 4 / 80^{+}$ cells were sorted by flow cytometry and used for subsequent studies.

Complete Freund's Adjuvant (CFA) Inflammation Model Fifty microliters of CFA was emulsified with $50 \mu \mathrm{l}$ of PBS, and the total of $100 \mu \mathrm{l}$ was injected subcutaneously under the dorsal skin. Ten days after injection, mice were killed, and their blood and spleen myeloid cells were analyzed.

\section{Statistics}

All data are expressed as the arithmetic means \pm s.e.m. Comparisons between two groups of animals or treatments were made by one-way ANOVA or by two-tailed Student's $T$-test using the software programs GraphPad Prism and Excel. Values of $P<0.05$ were considered statistically significant.

\section{RESULTS}

\section{Induction of MDSCs from BM In Vitro}

Culture of mouse BM with GM-CSF and either IL-6 or tumor-conditioned medium generates MDSCs. ${ }^{17,18}$ A 4-day culture of WT BM using either cell culture condition produced a mixed population of adherent and non-adherent cells. Analysis with trypan blue and 7-AAD showed that both populations were composed of living cells. The two populations were then separated, and each was co-cultured with stimulated $\mathrm{T}$ cells previously labeled with the fluorescent dye 5-(and 6)-carboxyfluorescein diacetate succinimidyl ester (Figure 1a). Our data showed that the co-culture of stimulated $\mathrm{T}$ cells with non-adherent BM cells suppressed T-cell proliferation, whereas an equivalent experiment in which adherent cells were co-cultured with stimulated $\mathrm{T}$ cells showed non-suppression. We also measured the surface markers of non-adherent and adherent cells and found significantly less surface expression of the maturation markers F4/80, CD80 and CD86 on non-adherent cells as compared with the adherent population (Figure 1b). Thus, the nonadherent cells are a relatively immature population capable of suppressing T-cell proliferation. Interestingly, the non-adherent cells express much less ACE than the adherent cell population (Figure 1c).

To investigate the differentiation potential of the non-adherent cells, they were cultured with the lineage growth factor macrophage colony-stimulating factor (M-CSF) to instruct differentiation to macrophages. Three days of culture upregulated the expression of F4/80, CD80 and CD86, indicating that the non-adherent cells are immature cells that can be induced to differentiate into macrophages (Supplementary Figure S1). In addition to increasing classical macrophage cell markers (ie, F4/80), culture with M-CSF also induced increased cell expression of ACE (Figure 1d).

MDSCs can be further subdivided based on their relative expression of the surface markers Ly6G and Ly6C. ${ }^{14}$ Cells with a $\mathrm{Ly}_{6 \mathrm{G}}{ }^{\text {low }} \mathrm{Ly} 6 \mathrm{C}^{\text {high }}$ phenotype are more closely aligned with monocytic lineage cells, while cells expressing
Ly6G $\mathrm{G}^{\text {high }}$ Ly6C $\mathrm{C}^{\text {low/ }}$ are more closely associated with a granulocytic-like morphology. To characterize the nonadherent cells found after a 4-day culture with GM-CSF and IL-6, the cells were analyzed for CD11b, Ly6G and Ly6C expression levels. More than $95 \%$ of the non-adherent cells were $\mathrm{CD} 11 \mathrm{~b}^{+}$. Using cell sorting, four groups of cells were isolated: $\mathrm{Ly}_{6 \mathrm{C}}{ }^{-} \mathrm{Ly}_{6 \mathrm{G}}{ }^{+}, \mathrm{Ly}_{6 \mathrm{C}}{ }^{+} \mathrm{Ly}_{6 \mathrm{G}}{ }^{+}, \mathrm{Ly}_{6 \mathrm{C}}{ }^{+} \mathrm{Ly}_{6} \mathrm{G}^{-}$and Ly6C ${ }^{-}$Ly6G $^{-}$(Figure 1e). Each population was assessed for its ability to suppress $\mathrm{T}$-cell proliferation. This showed that Ly6C ${ }^{+} \mathrm{Ly}_{6 \mathrm{G}}{ }^{+}$cells were the most potent suppressor cell population, while $\mathrm{Ly}_{6} \mathrm{C}^{-} \mathrm{Ly}_{6 \mathrm{G}}^{-}$cells were the weakest suppressors in this assay (Figure 1e). The $\mathrm{Ly}_{6} \mathrm{C}^{-} \mathrm{Ly}_{6 \mathrm{G}}{ }^{-}$population expressed the highest levels of surface MHC class II and CD86, suggesting that they are the most mature population among the non-adherent cells (Figure 1f). To investigate whether there are counterparts of $\mathrm{Ly}_{6 \mathrm{C}}{ }^{+} \mathrm{Ly}_{6 \mathrm{G}}{ }^{+}$cells in vivo, we examined the $\mathrm{BM}$, spleen and tumor tissues of mice with subcutaneous B16-F10 melanomas. The major composition of myeloid cells in the $\mathrm{BM}$ and spleen of tumor-bearing mice were Ly6 $\mathrm{C}^{\text {high }} \mathrm{Ly}_{6 \mathrm{G}}{ }^{-}$and Ly6 $\mathrm{C}^{\text {low }}$ Ly6 $\mathrm{G}^{\text {high }}$ cells (Supplementary Figure S2a). However, in the tumor tissue, we did find some $\mathrm{Ly}_{6 \mathrm{C}}{ }^{+} \mathrm{Ly}_{6 \mathrm{G}}{ }^{+}$myeloid cells (Supplementary Figure S2b).

To further investigate the $\mathrm{Ly}_{6 \mathrm{C}}{ }^{+} \mathrm{Ly}_{6 \mathrm{G}}{ }^{+}$cells, these cells were cultured with M-CSF for 3 days. Before culture, morphological examination showed that most of the $\mathrm{Ly} 6 \mathrm{C}^{+}$ Ly6G $^{+}$cells exhibited a granulocytic appearance. However, a 3-day culture with M-CSF converted most of the cells to a monocytic appearance (Supplementary Figures S3a and b). Also, the morphological change was accompanied by an increase of ACE expression (Supplementary Figure S3c).

\section{ACE Overexpression Reduces the In Vitro Generation of MDSCs}

To investigate the role of ACE in MDSC generation, we used BM from a mouse line termed ACE 10/10, which overexpresses ACE in myeloid cells. ${ }^{15}$ The culture of ACE 10/10 $\mathrm{BM}$ yielded comparable numbers of non-adherent cells as wild-type (WT) BM culture. However, in the non-adherent cell population, the ACE 10/10 cultured cells consistently yielded more of the relatively mature $\mathrm{Ly}_{6 \mathrm{C}}{ }^{-} \mathrm{Ly}_{6 \mathrm{G}}{ }^{-}$cells and fewer of the immunosuppressive $\mathrm{Ly}_{6 \mathrm{C}}{ }^{+} \mathrm{Ly} 6 \mathrm{G}^{+}$cells (Figure 2a). Further, detailed analysis of only the $\mathrm{Ly}_{6} \mathrm{C}^{+}$ ${\text { Ly } 6 \mathrm{G}^{+}}^{+}$cell population indicated that those cells derived from ACE 10/10 BM expressed higher levels of MHC class II (48\% increase in mean fluorescent intensity (MFI)) and CD86 (31\% increase in MFI) than equivalent cells derived from WT BM (Figure 2b). Thus, these data suggest that non-adherent cells derived from ACE 10/10 BM, a mouse line overexpressing ACE in myelomonocytic cells, appears somewhat more differentiated and less able to suppress T-cell proliferation than an equivalent population from WT BM (Figure 2c).

If ACE overexpression promotes monocytic differentiation, then this should be observed after the forced overexpression of ACE in WT BM cells. For this experiment, WT $\mathrm{BM}$ was transfected with either an expression vector encoding 
a
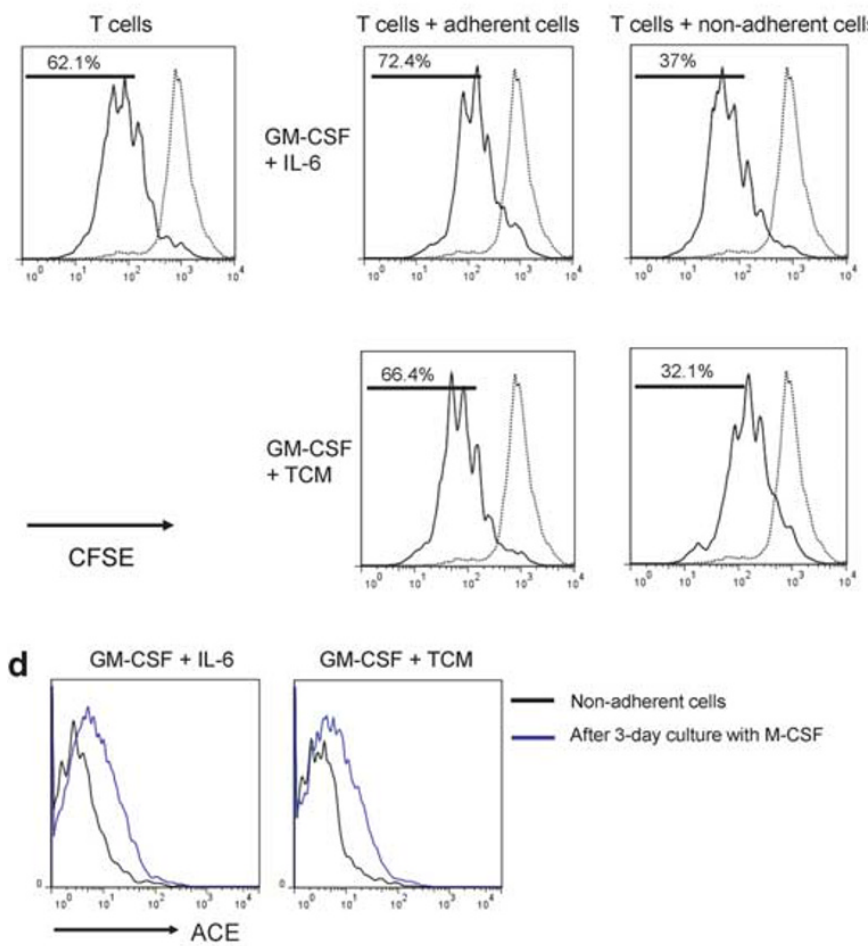

f
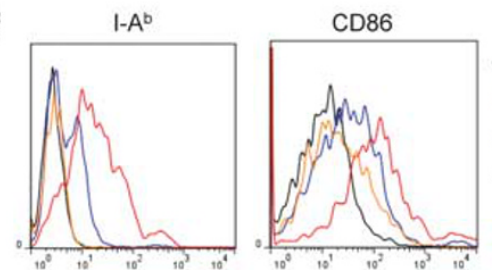

b

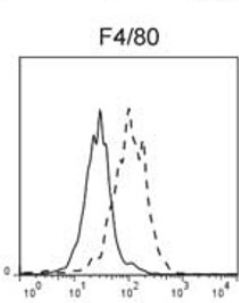

C
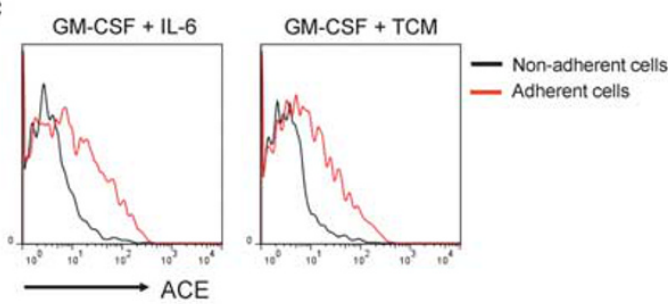

e

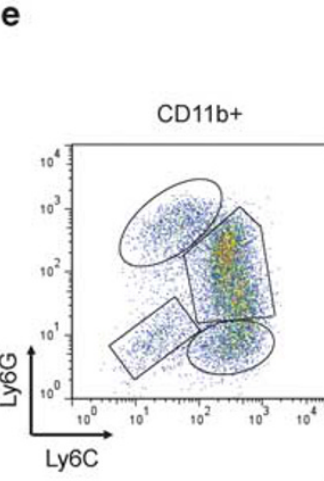

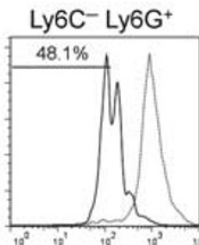
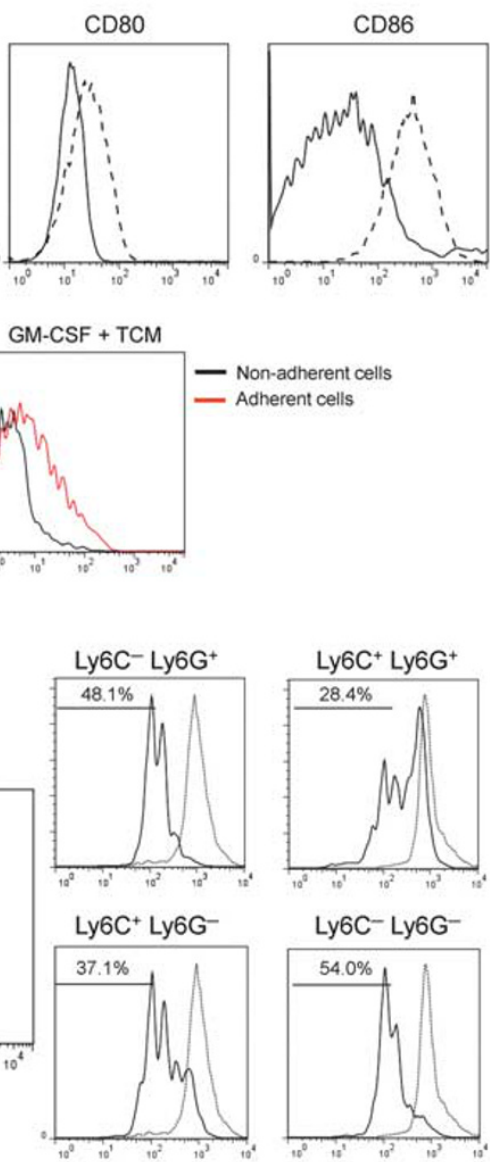

Adherent cells

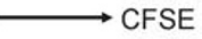

Figure 1 Myeloid-derived suppressor cell (MDSC) development in vitro. (a) Wild type (WT) bone marrow (BM) cells were cultured with granulocyte/ macrophage colony-stimulating factor (GM-CSF) and either IL- 6 or tumor-conditioned medium (TCM) for 4 days. The non-adherent and adherent cells were then harvested and separately co-cultured with anti-CD3- and anti-CD28-stimulated 5-(and 6)-carboxyfluorescein diacetate succinimidyl ester (CFSE)-labeled T cells for another 3 days. Typical T-cell proliferation profiles are shown (line) compared with the original T cells (dotted). The non-adherent cells suppress T-cell proliferation resulting in less dilution of CFSE. (b) The expression of surface maturation markers F4/80, CD80 and CD86 was assessed on the nonadherent and adherent cells. (c) ACE expression on the non-adherent and adherent cells. (d) ACE expression on the non-adherent cells with and without culture in macrophage-CSF (M-CSF). (e) The surface expression of Ly6G and Ly6C was measured on non-adherent cells (left). Four populations were identified: $\mathrm{Ly}_{6 \mathrm{C}}{ }^{-} \mathrm{Ly}_{6 \mathrm{G}}{ }^{+}, \mathrm{Ly}_{6 \mathrm{C}}{ }^{+} \mathrm{Ly}_{6 \mathrm{G}}{ }^{+}, \mathrm{Ly}_{6 \mathrm{C}}{ }^{+} \mathrm{Ly}_{6 \mathrm{G}}{ }^{-}$, and $\mathrm{Ly}_{6 \mathrm{C}}{ }^{-} \mathrm{Ly}_{6 \mathrm{G}}{ }^{-}$. These populations were gated and sorted, and their immunosuppressive activities were assessed by their ability to inhibit T-cell proliferation (right). (f) The surface maturation markers MHC class II (I-A $\mathrm{A}^{\mathrm{b}}$ ) and CD86 were evaluated on the gated cells. For all the experiments, the representative flow cytometry figures presented are representative of at least three independent experiments.

catalytically active ACE or a control vector expressing a fulllength but mutated form of ACE that is non-catalytic (mACE). ${ }^{19}$ Transfection by nucleofection was performed immediately upon isolation of BM cells from WT mice. After 4 days of culture, FACS analysis of ACE expression showed elevated and equivalent levels of surface ACE protein on the total floating cells transfected with either ACE or mACE (Supplementary Figure S4). Analysis of these cells showed that those transfected with WT ACE expressed more MHC class II (80\% increase in MFI) and CD86 (54\% increase in MFI) than cells transfected with the non-catalytic mACE (Figure 2d). Further, consistently less IL- $4 \mathrm{R} \alpha$ expression (64\% decrease in MFI) was expressed on the surface of ACEtransfected cells than cells transfected with mACE. As IL-4R $\alpha$ expression is a key feature of MDSCs, ${ }^{20}$ we assessed immunosuppression by co-incubating the transfected non-adherent cells with stimulated $\mathrm{T}$ cells. The $\mathrm{T}$ cells co-incubated with ACE-transfected BM culture proliferated one generation faster (ie, were less suppressed) than those co-incubated with mACE-transfected counterparts (Figure 2e). These data indicate that ACE expression not only promotes differentiation but also tilts myeloid cells away from an MDSC phenotype.

\section{ACE Overexpression in Myeloid Cells Induces a Pro-Inflammatory Phenotype and Reduced Tumor Growth} To study the role of ACE overexpression in myeloid maturation and tumor immunology, ACE 10/10 and WT mice 
a
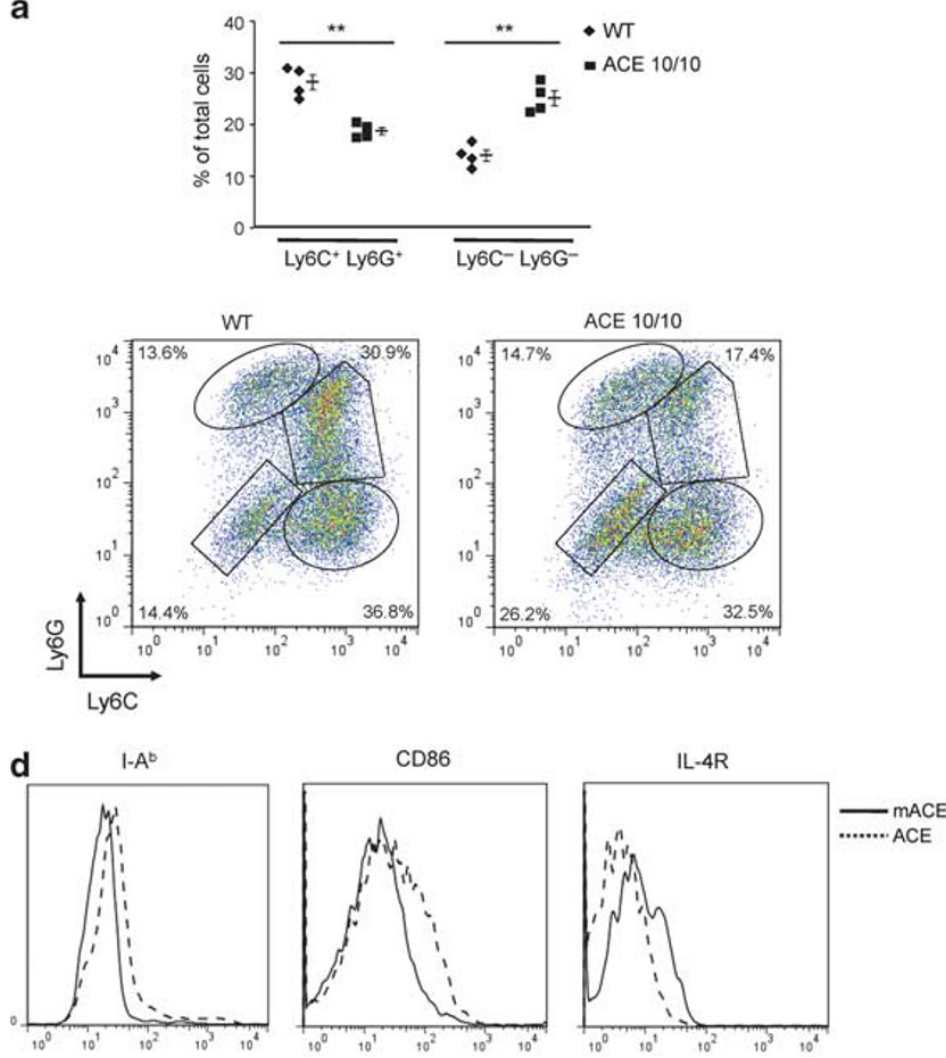
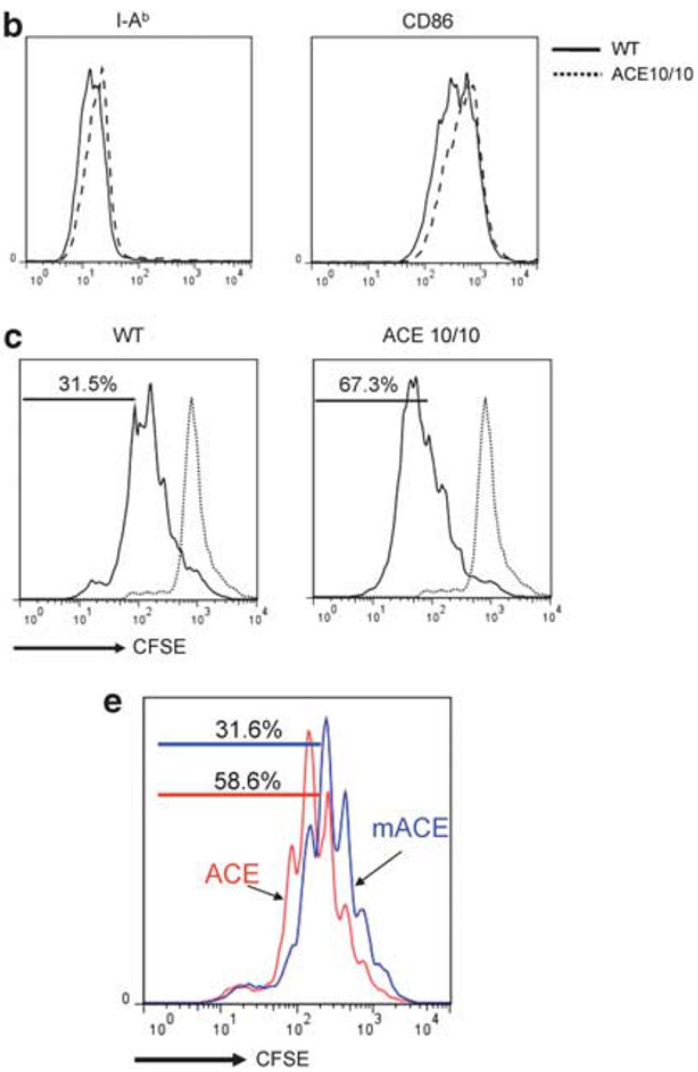

Figure 2 Angiotensin-converting enzyme (ACE) overexpression induces myeloid maturation. (a) The top portion of this panel shows the number of

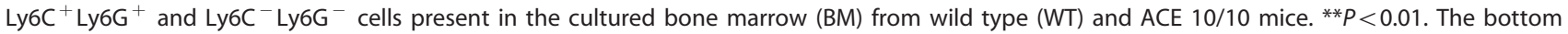
portion of this panel is a typical flow cytometric analysis of the WT and ACE 10/10 cells. (b) The expression levels of MHC class II and CD86 were measured on the surface of WT and ACE 10/10 BM-derived Ly6C ${ }^{+}$Ly6G ${ }^{+}$cells. (c) The non-adherent cells derived from WT and ACE 10/10 BM were co-incubated with stimulated 5-(and 6)-carboxyfluorescein diacetate succinimidyl ester (CFSE)-labeled T cells. Cytometric analysis after 3 days shows less suppression of T-cell proliferation in those cells co-cultured with the ACE 10/10 cells. (d) WT BM was transfected with vectors expressing either ACE or a catalytic domain mutated ACE (mACE). The surface expression of MHC class II, CD86 and IL-4R $\alpha$ was measured after a 4-day culture. (e) The non-adherent cells collected from ACE- or mACE-transfected BM cells were co-cultured with stimulated CFSE-labeled T cells. The T-cell proliferation profiles are presented and show less suppression of T-cell proliferation by those cells transfected with the catalytically active ACE construct. For all the experiments, the representative flow cytometry figures are typical of at least three independent experiments.

were challenged with B16-F10 melanoma cells by subcutaneous injection of tumor cells. Consistent with our previous data, ${ }^{15} 2$ weeks after B16 cell implantation, the resulting tumors in ACE 10/10 mice were significantly smaller (as determined by weight) than those in equivalently treated WT mice (ACE 10/10: $0.71 \pm 0.14 \mathrm{~g}$; WT: $1.38 \pm 0.13 \mathrm{~g}, P<0.001$, Figure 3a).

MDSC populations are thought capable of facilitating tumor metastases. Metastasis was evaluated in ACE 10/10 and WT mice by an i.v. injection of B16-F10 melanoma cells. After 2 weeks, the lungs were removed, and the number of visible metastases was counted (Figure 3b). Although WT lungs averaged 151 metastatic nodules, ACE 10/10 lungs averaged only 52 nodules $(P<0.01)$.

Above, we showed that ACE overexpression induced myeloid cell differentiation leading to the hypothesis that the enhanced anti-tumor response present in ACE 10/10 mice was, in part, due to reduced numbers of MDSCs.
To investigate this, ACE 10/10 and WT mice were challenged with subcutaneous injection of B16-F10 cells. After 2 weeks, animals were killed, and the number of MDSCs within the spleen was quantified by flow cytometry (Figure 3c). This showed lower numbers of $\mathrm{CD}_{11 \mathrm{~b}}{ }^{+} \mathrm{Gr} 1^{\text {intermediate(int) }}$ monocytic MDSCs in the spleens of ACE 10/10 tumorbearing mice. Specifically, 2 weeks after implantation of tumor cells, CD11b ${ }^{+} \mathrm{Gr} 1^{\text {int }}$ cells comprised only $4-8 \%$ of the cells in the ACE 10/10 spleens as compared with $8-15 \%$ of the cells in the spleens of WT tumor-bearing mice $(P<0.05)$. Further, ACE 10/10 mice treated with an ACE inhibitor captopril throughout the course of the tumor challenge had a consistent increase in the number of splenic $\mathrm{CD}_{11} \mathrm{~b}^{+} \mathrm{Gr} 1^{\mathrm{int}}$ cells. As $\mathrm{CD}_{11 \mathrm{~b}^{+}} \mathrm{Gr} \mathrm{l}^{\text {int }}$ cells are the most potent subpopulation of MDSCs, ${ }^{21-23}$ these data correlate with our previous published findings that treatment of ACE 10/10 mice with an ACE inhibitor increased the growth of tumor in this model. ${ }^{15}$ 

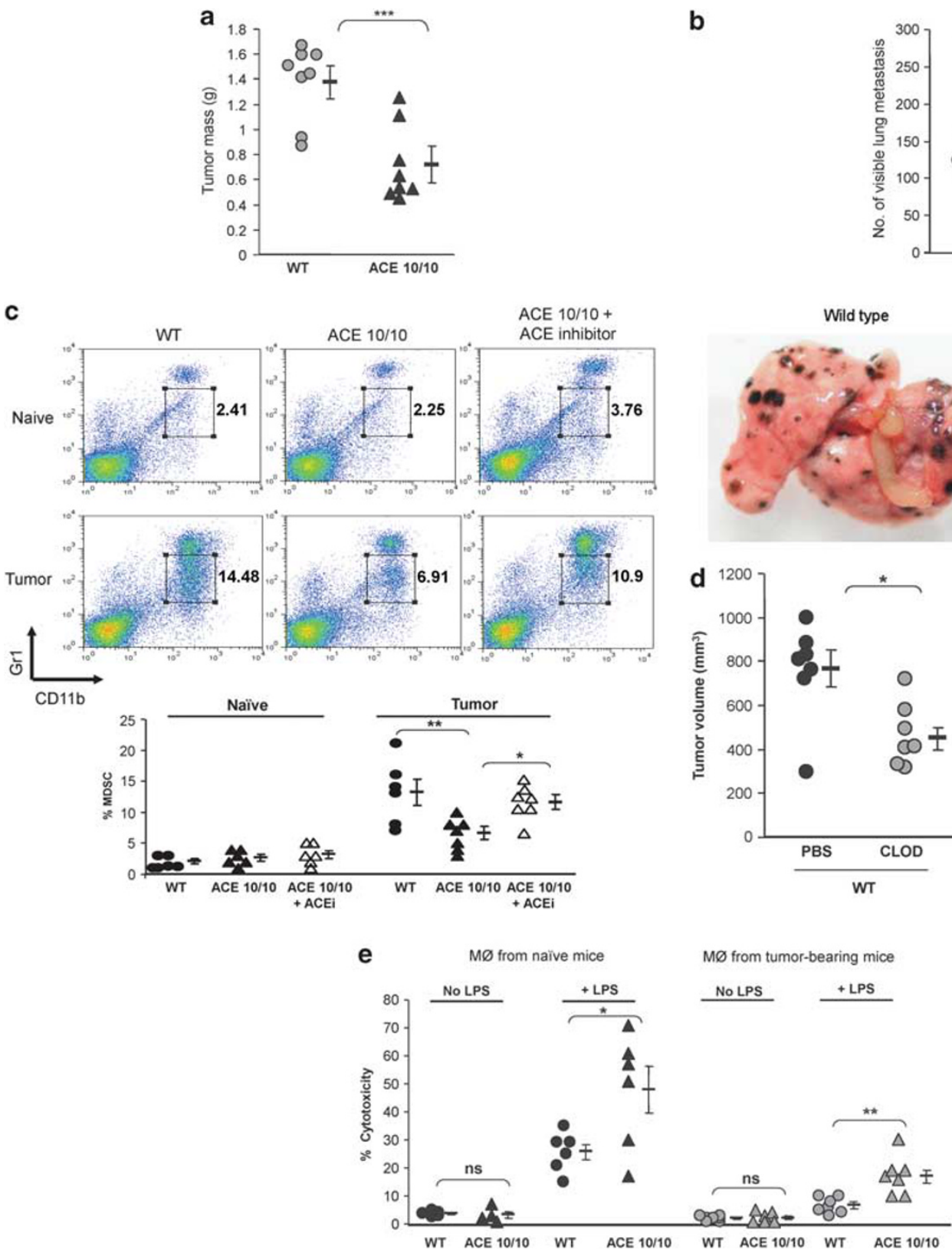

Figure 3 Angiotensin-converting enzyme (ACE) overexpression disrupts the myeloid-suppressing environment in cancer. (a) Wild type (WT) and ACE 10/10 mice were challenged intradermally with melanoma cells. Fourteen days later, tumors were dissected and weighed. Here and in panel (b), data from individual mice, as well as the group means and s.e.m., are shown. ${ }^{* * *} P<0.001$. (b) WT and ACE $10 / 10$ mice were challenged i.v. with $5 \times 10^{5}$ B16-F10 melanoma cells. Fourteen days later, metastatic nodules in the lungs were counted (top). ${ }^{*} P<0.02$. A representative photo of the lungs from a WT and ACE 10/10 mouse is shown (bottom). (c) Splenocytes from naive and tumor-bearing WT, ACE 10/10 and ACE 10/10 mice treated with the ACE inhibitor captopril were stained for CD11b and Gr1 and analyzed by flow cytometry. CD11 b ${ }^{+} \mathrm{Gr} 1^{\text {intermediate }}$ myeloid-derived suppressor cells (MDSCs) are indicated in the boxed area (top). The number of MDSCs from individual mice are shown (bottom), as well as the group means and s.e.m. ${ }^{*} P<0.05$, ${ }^{* *} P<0.01$. (d) Mice were depleted of macrophages by i.p. injection of liposome clodronate or liposome PBS for 1 week before tumor challenge and then every $72 \mathrm{~h}$. A group of ACE 10/10 mice were also treated with captopril. All mice were challenged with an intradermal injection of B16-F10. Tumor volume was measured on day 14. Although macrophage depletion reduces tumor size in WT mice, it results in larger tumors in ACE 10/10 mice. ${ }^{*} P<0.05,{ }^{* *} P<0.01,{ }^{* * *} P<0.001$. (e) Thioglycollate-elicited peritoneal macrophages or tumor-associated macrophages (TAM) were seeded in 96-well plates. Triplicate wells were primed overnight with $1 \mu \mathrm{g} / \mathrm{ml}$ lipopolysaccharide (LPS) or left untreated and then co-cultured with $2 \times 10^{4}$ tumor cells. Eighteen hours later, tumor cell death was evaluated by measuring LDH release into the supernatant. ${ }^{*} P<0.05$, ${ }^{*} P<0.02$, NS, not significant.

The above data suggest that ACE overexpression in monocytic cells tilts monocytic development away from a suppressive phenotype and toward a pro-inflammatory phenotype.
To further characterize the contribution of macrophage subsets in ACE 10/10 mice, we depleted monocytic myeloid cells using clodronate-encapsulated liposomes. ${ }^{24}$ Control 
groups were administered liposomes containing PBS. Flow cytometric analysis of blood confirmed that clodronateencapsulated liposomes effectively depleted monocytic myeloid cells equivalently in both ACE 10/10 and WT mice. In contrast, clodronate-PBS had no effect on these cells (Supplementary Figure S5). Consistent with previous reports, clodronate-encapsulated liposomes resulted in reduced tumor size by nearly $50 \%$ in WT mice (Figure $3 \mathrm{~d}$ ). These data confirm that in WT tumor-bearing mice, myeloid cells facilitate tumor growth. In contrast, depletion of monocytic myeloid cells in ACE 10/10 mice increased the tumor volume by nearly two-fold, indicating that myeloid cells expressing abundant ACE have a beneficial role in anti-tumor immunity. When ACE 10/10 mice were treated with the ACE inhibitor, tumor growth increased, implicating the enzymatic activity of ACE as being important in the myeloid-mediated anti-tumor effect present in this model (Figure 3d).

We posit that ACE overexpression changes the developmental phenotype of myelomonocytic cells, inducing differentiation and an effective pro-inflammatory response. To directly compare the ability of ACE 10/10 and WT macrophages to limit tumor, we established an in vitro tumor killing assay in which peritoneal macrophages from WT and ACE 10/10 mice were co-cultured with B16-F10 melanoma cells (Figure 3e, left). In the absence of macrophage activation by LPS, little killing of tumor cells was observed. However, LPS-stimulated peritoneal macrophages can kill tumor cells, and we observed that the ACE 10/10 macrophages killed tumor cells more efficiently than equivalent cells from WT mice (ACE 10/10: 48\% lysis; WT: 25\%, $P<0.05$ ). We also tested the ability of macrophages from tumor-bearing mice to directly kill tumor cells (Figure 3e, right). Cells from the peritoneal lavage of tumor-bearing mice were cultured, and the macrophages isolated from WT mice killed tumor cells less effectively than equivalent cells from ACE 10/10 animals (7 vs $24 \%, P<0.02$ ). Together, these data indicate that the increased expression of ACE in myeloid cells skews myeloid differentiation to a more pro-inflammatory phenotype. This was consistently true in both tumor and non-tumor bearing mice.

\section{ACE Expression is Inversely Associated with the Development of MDSCs}

A potential caveat in analyzing MDSCs in tumor-bearing mice is that often MDSC development correlates with tumor size. Because of this, we also studied MDSC development in the setting of chronic inflammation caused by challenging mice with CFA. CFA is an emulsified solution containing killed mycobacteria and is very effective in stimulating chronic inflammation. Subcutaneous CFA has been found to reproducibly induce MDSC development. ${ }^{6,12,25}$

ACE 10/10, WT and ACE-null (knockout or KO) ${ }^{16}$ mice were analyzed under basal conditions and after challenge with CFA (Figure 4a). Under basal conditions, there was no difference between ACE 10/10 and WT mice in the number of $\mathrm{CD}_{11 b^{+}} \mathrm{Grl}^{+}$cells in either the blood or the spleen. However, as we previously showed, ACE KO mice do have a small but significant increase in these cells in both the blood and spleen. ${ }^{3}$ In all the groups, levels of MDSCs $\left(\mathrm{CD} 11 \mathrm{~b}^{+} \mathrm{Gr} 1^{+}\right)$
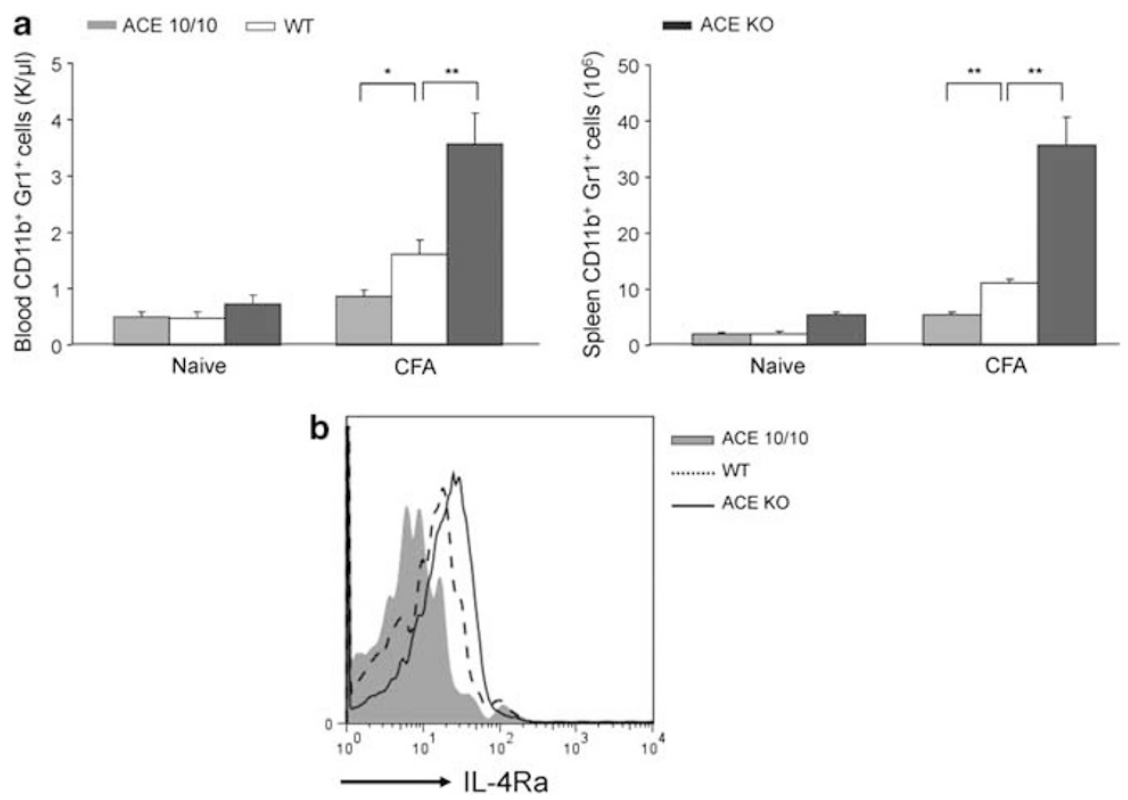

Figure 4 Myeloid Angiotensin-converting enzyme (ACE) expression is inversely correlated with myeloid-derived suppressor cells (MDSCs) developed in inflammation. (a) ACE 10/10, WT and ACE KO mice were challenged with emulsified complete Freund's adjuvant (CFA) subcutaneously. Ten days later, the blood and spleen were collected, and the number of $\mathrm{CD} 11 \mathrm{~b}^{+} \mathrm{Gr}^{+}{ }^{+}$cells was determined. ${ }^{*} P<0.05,{ }^{*} P<0.01, n=5$. (b) The IL-4R $\alpha$ expression on the splenic $\mathrm{CD} 11 \mathrm{~b}^{+} \mathrm{Gr} 1^{+}$cells was measured. The figure is representative of at least four separate mice in each group. 
cells markedly change after immunization with CFA; all the groups show an increased number of these cells. However, there is a marked difference depending on the expression levels of ACE. For example, the spleens of CFA-treated ACE $10 / 10$ mice averaged $5.4 \times 10^{6}$ MDSCs, compared with equivalently treated WT mice, which have $11 \times 10^{6}$, and ACE KO mice, which show $>35 \times 10^{6}$ splenic MDSCs. Not only do ACE 10/10 mice challenged with CFA produce fewer MDSCs, but even the biochemical details of the $\mathrm{CD} 11 \mathrm{~b}^{+} \mathrm{Gr}^{+}$cells are different from WT or ACE KO cells. Flow cytometric analysis of these cells for expression of the immunosuppressive marker IL- $4 \mathrm{R} \alpha$ showed significant differences between cells from the three groups of mice

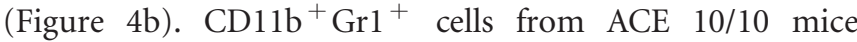
expressed lesser amounts of IL-4R $\alpha$ (68\% decreased MFI) than equivalent cells from WT mice. In contrast, ACE KO mice increased IL-4R $\alpha$ ( $47 \%$ increase of MFI) as compared with WT. Thus, analysis of MDSCs induced by CFA is consistent with the conclusions derived from study of tumorinduced MDSCs: ACE expression facilitates myeloid maturation and inhibits the development of MDSCs.

\section{DISCUSSION}

Monocytic cells are key sentinels of the innate immune system and important antigen-presenting cells required for adaptive immune responses. Although macrophages and other myeloid cells are heavily recruited within the tumor microenvironment, they are ineffectual. In fact, in both animal models and humans, MDSCs may, in concert with the tumor stroma, promote tumor growth and the escape of tumor from host T-cell responses. ${ }^{26}$ It is thought that MDSCs originate from defective myelopoiesis that results in a heterogeneous group of myeloid cells in an immature state of differentiation. ${ }^{6}$ These tumor-associated myeloid cells have an altered transcriptional program, including the downregulation of a number of factors critical for eliciting an appropriate pro-inflammatory response. ${ }^{27}$

In ACE 10/10 mice, ACE is overexpressed in myelomonocytic lineage cells. These mice markedly resist melanoma and lymphoma ${ }^{15}$ and have better tumor control in both orthotopic and i.v. metastatic models. However, there are caveats. Whether ACE expression in myeloid cells is universally associated with less tumor metastasis needs further examination using spontaneous metastatic models. Also, any conclusion in mice has to be examined in other, more clinically relevant models. Previous histological analysis showed that melanoma in ACE $10 / 10$ mice contain substantially increased numbers of inflammatory myeloid cells and that this is associated with an increased number of anti-tumor T cells. ${ }^{15}$ Here, we show that there is significantly lower numbers of MDSCs in tumor-bearing ACE 10/10 mice. In fact, myeloid cells appear to be pro-inflammatory, as indicated by increased in vitro tumor-killing activity. The effect of this change in phenotype was demonstrated by using clodronate to eliminate monocytic cells in both WT and ACE
10/10 mice. In WT mice, monocytic cells appear to facilitate tumor growth; elimination of such cells resulted in a smaller tumor size. In contrast, monocytic cells in the ACE 10/10 appear to have an overall anti-tumor effect, as their elimination was associated with increased tumor growth. Thus, we posited that ACE overexpression skews the myeloid cells in the tumor environment to a more pro-inflammatory phenotype associated with fewer anti-inflammatory MDSCs.

MDSCs consist of immature cells and have the potential to differentiate and mature to other myeloid cells in response to local signals. For example, hypoxia in the tumor microenvironment upregulates the transcription factor HIF1 $\alpha$, which drives the differentiation of MDSCs to macrophages; ${ }^{28}$ published results also show that a large number of monocytic MDSCs in tumor-bearing mice eventually obtain the features of granulocytic MDSCs. ${ }^{29}$ We now present several lines of evidence indicating that ACE expression in monocytic cells leads to increased maturation associated with an increased pro-inflammatory phenotype. First, MDSCs expressed significantly lower levels of ACE compared with the macrophages developed in the same BM culture dish. Moreover, ACE was upregulated when the MDSCs differentiated to macrophages under the induction of M-CSF, and ACE upregulation correlated with the upregulation of the maturation markers CD80, CD86 and MHC class II. Second, when we forced ACE overexpression in WT BM, the BM developed more mature myeloid cells compared with BM overexpressing a catalytically inactive ACE. These data suggest that ACE expression may directly contribute to myeloid maturation. Consistent with this idea, we previously reported that the expression of ACE is increased when monocytes differentiate to macrophages and can be further upregulated when macrophages and dendritic cells are activated by the pro-inflammatory cytokine IFN- $\gamma$. Further, listeria infection significantly upregulated ACE in splenic macrophages and dendritic cells but not in lymphocytes. ${ }^{19}$ Finally, using ACE KO, WT and ACE 10/10 mice in the CFA model, we showed that there was an inverse relationship between ACE expression and the developmental phenotype of monocytic cells; the absence of ACE is associated with significant developmental immaturity of monocytic cells, whereas ACE 10/10 mice, which overexpress ACE in myelomonocytic cells, have less MDSCs and an enhanced immune response.

Myelopoiesis is a fine-tuned process in that a minor disruption can result in severe consequences, as myeloid cells are in the first-line of anti-bacterial defense and clearance. Our work suggests that during tumor growth and other inflammatory settings, myelomonocytic cells modified to overexpress ACE are better suited to maintain a relatively mature pro-inflammatory phenotype, which is unfavorable to the development of immature MDSCs. Considering ACE is a peptidase, a next step may be to investigate which bioactive peptide(s) mediates the function of ACE on MDSC development. Such an exact identification might make it feasible to clinically manipulate MDSC development in the 
future. This research also raises the question of whether ACE inhibition in humans changes myeloid cell populations.

Supplementary Information accompanies the paper on the Laboratory Investigation website (http://www.laboratoryinvestigation.org)

\section{ACKNOWLEDGMENTS}

This work was supported by an AHA Grant 13BGIA14680069 (to XZS) and NIH Grants R01 HL110353-26 (to KEB) and F32 HL105036 (to FSO).

\section{DISCLOSURE/CONFLICT OF INTEREST}

The authors declare no conflict of interest.

1. Shen $X Z$, Ong FS, Bernstein EA, et al. Nontraditional roles of angiotensin-converting enzyme. Hypertension 2012;59:763-768.

2. Platten $M$, Youssef $S$, Hur EM, et al. Blocking angiotensin-converting enzyme induces potent regulatory $\mathrm{T}$ cells and modulates $\mathrm{TH} 1-$ and TH17-mediated autoimmunity. Proc Natl Acad Sci USA 2009;106: 14948-14953.

3. Lin C, Datta V, Okwan-Duodu D, et al. Angiotensin-converting enzyme is required for normal myelopoiesis. FASEB J 2011;25:1145-1155.

4. Bernstein KE, Ong FS, Blackwell WL, et al. A modern understanding of the traditional and nontraditional biological functions of angiotensinconverting enzyme. Pharmacol Rev 2013;65:1-46.

5. Hori S, Nomura T, Sakaguchi S. Control of regulatory $T$ cell development by the transcription factor Foxp3. Science 2003;299: 1057-1061.

6. Gabrilovich DI, Nagaraj S. Myeloid-derived suppressor cells as regulators of the immune system. Nat Rev Immunol 2009;9:162-174.

7. Otsuji $M$, Kimura $Y$, Aoe $T$, et al. Oxidative stress by tumor-derived macrophages suppresses the expression of $\mathrm{CD} 3$ zeta chain of T-cell receptor complex and antigen-specific T-cell responses. Proc Natl Acad Sci USA 1996;93:13119-13124.

8. Pak AS, Wright MA, Matthews JP, et al. Mechanisms of immune suppression in patients with head and neck cancer: presence of CD34( + ) cells which suppress immune functions within cancers that secrete granulocyte-macrophage colony-stimulating factor. Clin Cancer Res 1995;1:95-103.

9. Marigo I, Dolcetti $L$, Serafini $P$, et al. Tumor-induced tolerance and immune suppression by myeloid derived suppressor cells. Immunol Rev 2008;222:162-179.

10. Delano MJ, Scumpia PO, Weinstein JS, et al. MyD88-dependent expansion of an immature GR-1 $(+) \mathrm{CD} 11 \mathrm{~b}(+)$ population induces $\mathrm{T}$ cell suppression and Th2 polarization in sepsis. J Exp Med 2007;204:1463-1474.

11. Munera V, Popovic PJ, Bryk J, et al. Stat 6-dependent induction of myeloid derived suppressor cells after physical injury regulates nitric oxide response to endotoxin. Ann Surg 2010;251:120-126.

12. Pastor $\mathrm{S}$, Minguela $\mathrm{A}, \mathrm{Mi} \mathrm{W}$, et al. Autoantigen immunization at different sites reveals a role for anti-inflammatory effects of
IFN-gamma in regulating susceptibility to experimental autoimmune encephalomyelitis. J Immunol 2009;182:5268-5275.

13. Gabrilovich DI, Velders MP, Sotomayor EM, et al. Mechanism of immune dysfunction in cancer mediated by immature Gr-1 + myeloid cells. J Immunol 2001;166:5398-5406.

14. Youn Jl, Gabrilovich DI. The biology of myeloid-derived suppressor cells: the blessing and the curse of morphological and functional heterogeneity. Eur J Immunol 2010;40:2969-2975.

15. Shen XZ, Li P, Weiss $D$, et al. Mice with enhanced macrophage angiotensin-converting enzyme are resistant to melanoma. Am J Pathol 2007;170:2122-2134.

16. Esther $\mathrm{CR}$, Howard TE, Marino EM, et al. Mice lacking angiotensinconverting enzyme have low blood pressure, renal pathology, and reduced male fertility. Lab Invest 1996;74:953-965.

17. Marigo I, Bosio E, Solito S, et al. Tumor-induced tolerance and immune suppression depend on the C/EBPbeta transcription factor. Immunity 2010;32:790-802.

18. Youn Jl, Nagaraj S, Collazo $\mathrm{M}$, et al. Subsets of myeloid-derived suppressor cells in tumor-bearing mice. J Immunol 2008;181: $5791-5802$.

19. Shen $X Z$, Billet $S$, Lin $C$, et al. The carboxypeptidase ACE shapes the MHC class I peptide repertoire. Nat Immunol 2011;12:1078-1085.

20. Roth F, De La Fuente AC, Vella JL, et al. Aptamer-mediated blockade of IL4R $\alpha$ triggers apoptosis of MDSCs and limits tumor progression. Cancer Res 2012;72:1373-1383.

21. Priceman SJ, Sung JL, Shaposhnik Z, et al. Targeting distinct tumor-infiltrating myeloid cells by inhibiting CSF-1 receptor: combating tumor evasion of antiangiogenic therapy. Blood 2010;115: 1461-1471.

22. Movahedi K, Guilliams M, Van den Bossche J, et al. Identification of discrete tumor-induced myeloid-derived suppressor cell subpopulations with distinct T cell-suppressive activity. Blood 2008;111: 4233-4244.

23. Dolcetti L, Peranzoni E, Ugel S, et al. Hierarchy of immunosuppressive strength among myeloid-derived suppressor cell subsets is determined by GM-CSF. Eur J Immunol 2010;40:22-35.

24. Van Rooijen N, Sanders A. Liposome mediated depletion of macrophages: mechanism of action, preparation of liposomes and applications. J Immunol Methods 1994;174:83-93.

25. Cheng $\mathrm{P}$, Corzo CA, Luetteke $\mathrm{N}$, et al. Inhibition of dendritic cell differentiation and accumulation of myeloid-derived suppressor cells in cancer is regulated by S100A9 protein. J Exp Med 2008;205: 2235-2249.

26. Qian BZ, Pollard JW. Macrophage diversity enhances tumor progression and metastasis. Cell 2010;141:39-51.

27. Sonda N, Chioda M, Zilio S, et al. Transcription factors in myeloidderived suppressor cell recruitment and function. Curr Opin Immunol 2011:23:279-285.

28. Corzo CA, Condamine T, Lu L, et al. HIF- $1 \alpha$ regulates function and differentiation of myeloid-derived suppressor cells in the tumor microenvironment. J Exp Med 2010;207:2439-2453.

29. Youn Jl, Kumar V, Collazo $M$, et al. Epigenetic silencing of retinoblastoma gene regulates pathologic differentiation of myeloid cells in cancer. Nat Immunol 2013;14:211-220. 